\title{
High intensity neutrino beams
}

$\operatorname{AUTHOR}(\mathrm{S}):$

Ichikawa, A. K.

\section{CITATION:}

Ichikawa, A. K.. High intensity neutrino beams. AIP Conference Proceedings 2015, 1666(1): 130001.

\section{ISSUE DATE:}

2015-07-15

URL:

http://hdl.handle.net/2433/230146

\section{RIGHT:}

(C) 2015 AIP Publishing LLC. This article may be downloaded for personal use only. Any other use requires prior permission of the author and AIP Publishing. The following article appeared in 'A. K. Ichikawa, High intensity neutrino beams, AIP Conference Proceedings 1666, 130001 (2015)' and may be found at

https://aip.scitation.org/doi/abs/10.1063/1.4915579.; The full-text file will be made open to the public on 15 July 2016 in accordance with publisher's 'Terms and Conditions for Self-Archiving' 


\section{High intensity neutrino beams}

A. K. Ichikawa

Citation: AIP Conference Proceedings 1666, 130001 (2015); doi: 10.1063/1.4915579

View online: https://doi.org/10.1063/1.4915579

View Table of Contents: http://aip.scitation.org/toc/apc/1666/1

Published by the American Institute of Physics

\section{Articles you may be interested in}

Neutrino cross-sections: Experiments

AIP Conference Proceedings 1666, 060003 (2015); 10.1063/1.4915559

Recent developments in neutrino-nucleus interactions in $1 \mathrm{GeV}$ energy region

AIP Conference Proceedings 1666, 060001 (2015); 10.1063/1.4915557

Conference Photo: XXVI International Conference on Neutrino Physics and Astrophysics

AIP Conference Proceedings 1666, 010003 (2015); 10.1063/1.4916192

Discovering the Majorana neutrino: The next generation of experiments

AIP Conference Proceedings 1666, 170005 (2015); 10.1063/1.4915595 


\title{
High Intensity Neutrino Beams
}

\author{
A. K. Ichikawa \\ Department of Physics, Faculty of Science, Kyoto University, Kyoto, 606-8502, Japan
}

\begin{abstract}
High-intensity proton accelerator complex enabled long baseline neutrino oscillation experiments with a precisely controlled neutrino beam. The beam power so far achieved is a few hundred $\mathrm{kW}$ with enourmorous efforts of accelerator physicists and engineers. However, to fully understand the lepton mixing structure, MW-class accelerators are desired. We describe the current intensity-frontier high-energy proton accelerators, their plans to go beyond and technical challenges in the neutrino beamline facilities.
\end{abstract}

Keywords: neutrino, accelerator

\section{INTRODUCTION}

Growing interests on the unresolved problems of the neutrino oscillation can only be satisfied with the realization of the high intensity neutrino beam. In this paper, we present what is the limitting factor of intensity and how people are trying to overcome those. Here, we restrict the topics to the conventional neutrino beam, which have been used for accelerator-based long baseline neutrino oscillation experiments.

Figure 1 shows a typical configuration of accelerator-based long basline neutrino experiments. A proton beam from an acelerator-complex is injected to a target. Pions and Kaons are generated there, are focused forward by electromagnetic horns and eventually decay mainly into muons and muon-neutrinos after a few hundred to a few thorusand metors flight. Those neutrinos are detected at near detectors on accelerator cite and at a far detector located a few handred to a few thoutand km away. As an example to demonstrate the intensity of these neutrino beams, when J-PARC, which is described later, run at the design power of $750 \mathrm{~kW}$, about $1 \mathrm{v} / \mathrm{cm}^{2} / \mathrm{s}$ reaches the $295 \mathrm{~km}$ away far detector.

The produced neutrino beam has following features:

- wide band spectrum, but can be narrowed by changing the target-horn distance (NuMI) or by the off-axis method (T2K, NOvA) as shown in Fig.2,

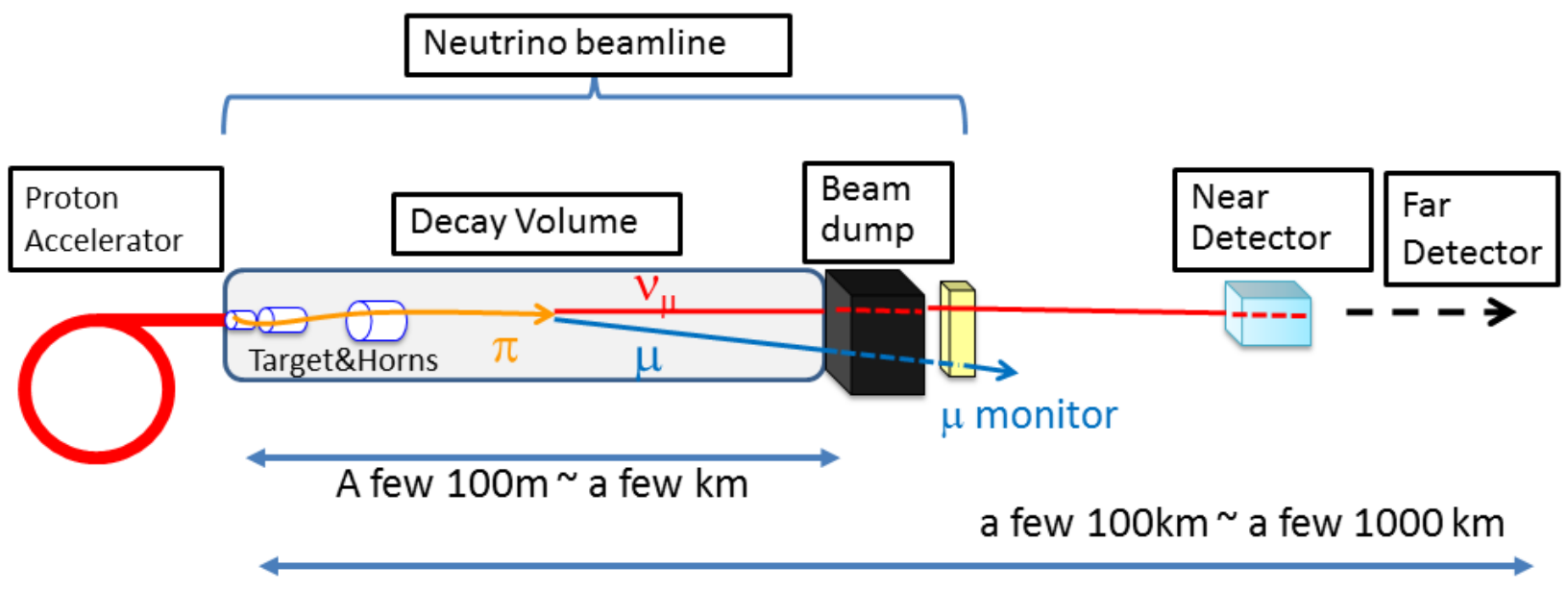

FIGURE 1. Components of the accelerator neutrino experiment 

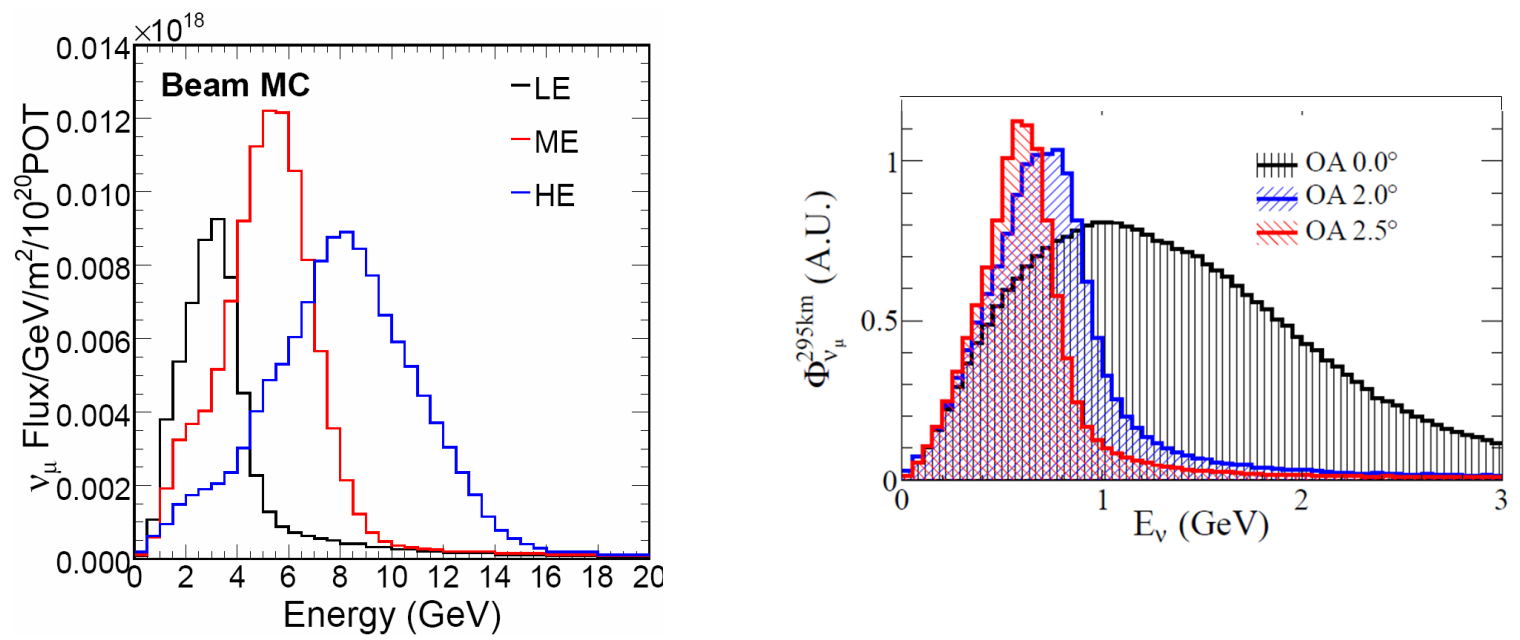

FIGURE 2. Neutrino beam spectra at NuMI[1] for various target-horn configurations (left) and J-PARC for various off-axis angle settings(right).

- a few $\% v_{e}$ contamination from the decay of Kaons and muons,

- $v$ beam or $\bar{v}$ beam is produced depending on the horn current polarity,

- size of the uncertainty mainly arise from the uncertainty of the hadron production by the $p$-nucleus interaction.

We first describe the current intensity-frontier high-energy proton accelerators and plans to go beyond. Then, we show the technical challenges in the neutrino beamline facilities.

\section{INTENSITY FRONTIER ACCELERATORS}

Three laboratories, distributed in three regions of Europe, Japan and US, have high-energy proton accelerators for neutrino experiments. Those are summarized in Table 1 together with the achieved and planned beam intensities in terms of power. Beam power is calculated by multiplying the beam energy, number of protons per pulse and acceleration repetition rate, and is a good measure both for neutrino beam intensity and facility capability.

TABLE 1. Intensity frontier machine for neutrino experiments

\begin{tabular}{lcccc}
\hline & \multirow{4}{*}{ Energy } & \multicolumn{3}{c}{ Power (MW) } \\
\cline { 3 - 5 } & & current & planned & future \\
\hline J-PARC/KEK Main Ring & $30 \mathrm{GeV}$ & $\sim 0.25 \mathrm{MW}$ (T2K) & 0.75 (T2K) & $\sim 2$ \\
FNAL NuMI & $120 \mathrm{GeV}$ & $\sim 0.36 \mathrm{MW}$ (MINOS) & 0.7 (NOvA) & $\sim 2$ (LBNF) \\
CERN SPS & $400 \mathrm{GeV} / c$ & $0.3 \sim 0.5 \mathrm{MW}$ (OPERA/ICARUS) & $(0.2$ for CENF) & $0.7 \sim 2$ (CN2PY) \\
\hline
\end{tabular}

Before describing intensity upgrade plans of each facility in the following sub-sections, it would be worth to mention here about the one of the key issues at the intensity frontier machine: the space-charge effect. It is caused by the repulsive forces between beam particles and results in blow-up of the beam. The effect is larger at lower energy and increase non-linearity with the beam intensity. It causes the beam loss during the acceleration and limit the intensity of the machine. Possible solutions to overcome this are to increase repetition rate without increasing protons-perpulse(PPP) and to increase the injection energy. Usage of linac-only acceleration, one path accelerator, can drastically reduce the limitation, but have difficulty to go to high energy.

\section{J-PARC}

J-PARC - Japan Proton Accelerator Complex - consists of a $400 \mathrm{MeV}$ linac, a $3 \mathrm{GeV}$ Rapid-Cycle Synchrotron (RCS) and a $30 \mathrm{GeV}$ Main Ring synchrotron (MR) as shown in Fig.3(a). A neutrino beam is produced by using the 


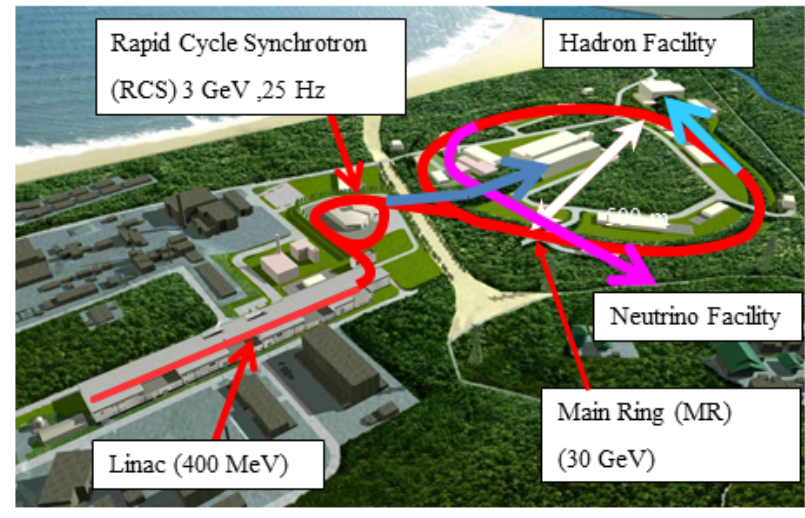

(a) J-PARC

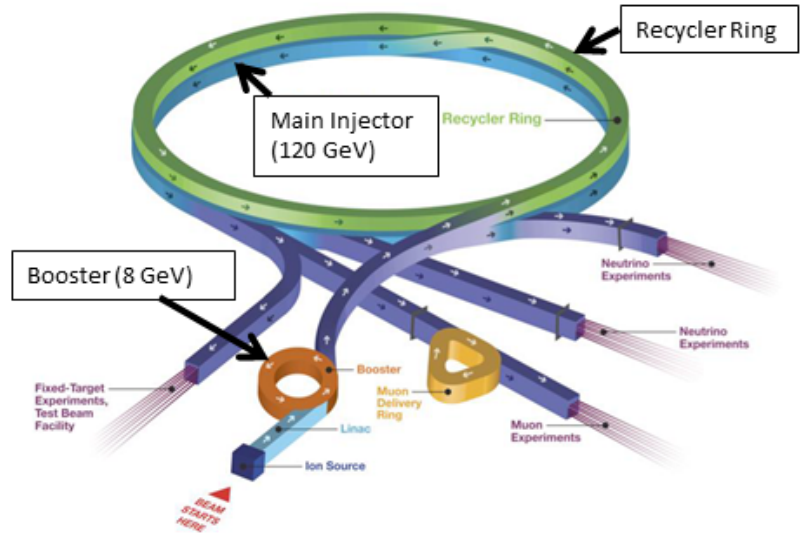

(b) Fermilab Accelerator Complex

FIGURE 3. Accelerator complexes at J-PARC and Fermilab.

proton beam from MR. The intensity is now limited to about $0.25 \mathrm{MW}$ because of the beam loss at the injection to MR. The proton-per-pulse already recorded world highest number and it is difficult to significantly increase it. To achieve the design intensity of $0.75 \mathrm{MW}$ by 2017 , it is planed to increase the repetition rate of the MR acceleration cycle from $1 / 2.5 \mathrm{~Hz}$ to $1.3 \mathrm{~Hz}$ by replacing magnet power supplies and RF cavities. The new RF cavity having high impedance was already developed and the the new magnet power supply development is on-going. For more future, a new $8 \mathrm{GeV}$ Booster synchrotron between RCS and MR is being discussed. The target in that case is $1 \sim 3 \mathrm{MW}$.

\section{FNAL}

The Fermilab proton accelerator complex, as shown in Fig.3(b), consists of a $400 \mathrm{MeV}$ linac, a $8 \mathrm{GeV}$ booster, a recycler ring (RR) and a main injector (MI). The current MI intensity is $280 \mathrm{~kW}$ and limited by the booster intensity. Many attempts have been made to surmount the situation. The MI acceleration cycle was shortened with faster ramp. Each of MI buckets is filled twice by two booster batches by the method 'slip stacking'. RR is a ring made of permanent magnets and can store a $8 \mathrm{GeV}$ beam. It was originally build to store the anti-proton beam for Tevatron, but now is used to store the beam from the booster while MI is in acceleration period. Once MI becomes ready to accept the new beam, the beam stored in RR is injected. This scheme reduce the time required for the beam injection compared to the case when the beam is directly injected from the booster. With the combination of all these efforts including the slip stacking from the booster to RR, it is planned to increase the MI beam intensity up to $700 \mathrm{~kW}$ by the end of 2015 .

In further future, FNAL has an power upgrade plan called 'Proton Improvement Plan (PIP) II'. The goal is to achieve $1 \mathrm{MW}$ until 2025. The booster intensity will be increased by a higher energy beam from a new $800 \mathrm{MeV}$ super-conducting pulsed linac.

\section{Europe}

In Europe, the CN2PY (CERN-to-Pyhasalmi) long baseline neutrino oscillation project is being discussed. Initial phase of this project will use a $750 \mathrm{~kW} 400 \mathrm{GeV}$ beam from the existing SPS (Super-Proton-Synchrotron). A possible option for the second phase of the project is a $2 \mathrm{MW} 50 \mathrm{GeV}$ beam by the combination of a new proton Synchrotron, HP-PS and a $4 \mathrm{GeV}$ superconducting linac, LP-SPL.

Another proposal from Europe is to utilize ESS, European Spallation Source in Sweden. It is a $2 \mathrm{GeV} 5 \mathrm{MW}$ superconducting linac expected to start in 2019. To use the beam from ESS for a neutrino experiment, it is proposed to increase the beam power from $5 \mathrm{MW}$ to $10 \mathrm{MW}$ by increasing the frequency of the linac from $14 \mathrm{~Hz}$ to $28 \mathrm{~Hz}$. 


\section{TECHNICAL CHALLENGES IN NEUTRINO BEAM FACILITY}

After the acceleration, the proton beam is extracted to the neutrino beam facility, where it is injected to a target material and produce pions and Kaons which eventually decay into neutrinos. There are many technical challenges to accept a high intensity proton beam and to produce a high intensity neutrino beam: beam loss in the primary beamline, thermal shock, radiation damage, cooling issue, dirty product in air and water, radiation shielding, and so on.

When a proton beam hits material, energy is deposited into material and the temperature increases. Since the proton beam is delivered as short pulses from the accelerator, temperature rise happens while there is no time for the material to expand. This causes the thermal shock stress in the material. With $\sim 1 \mathrm{MW} \sim 1 \mathrm{~Hz}$ beam, solid materials denser than Titanium would be destroyed by this thermal shock. Therefore, the target and beam window are usually made with material lighter than Titanium.

With high irradiation, any high-molecular material cannot withstand. As an example, $25 \%$ of atoms in the graphite target will be displaced by beam radiation with one year $0.75 \mathrm{MW}$ operation at J-PARC. The material properties are changed with such environment. The $90 \mathrm{~cm}$ long graphite target is expected to shrink by $\sim 5 \mathrm{~mm}$ in length and its thermal conductivity may be decreased by $70 \sim 95 \%$ with five year $0.75 \mathrm{MW}$ operation at J-PARC.

The power of the primary proton beam is deposited in the target and horns, and also in the decay-volume wall and beam dump. Therefore, larger amount of cooling water or gas is necessary for higher beam power. Hazardous products such as Tritium, $\mathrm{H}_{2}$, NOx and acid are produced from these medium by irradiation. Carefully treatment and efforts of reduction of these products have to be developed.

\section{Target}

\section{T2K target}

The T2K target is made of a graphite rod of $26 \mathrm{~mm}$ in diameter and $910 \mathrm{~mm}$ in length, whose schematic view is shown in Fig. 4. It was designed for $0.75 \mathrm{MW}$ beam. To avoid the water hammer at the beam incident, Helium-gas cooling was adopted. The rod is surrounded by another graphite tube and contained in a Titanium-alloy container. These make the Helium-gas flow. The cooling by Helium-gas enables the operation with a temperature as high as $700{ }^{\circ} \mathrm{C}$ and reduce the material-property change due to radiation. The maximum instantaneous temperature rise at the beam incident is about $200 \mathrm{~K}$ and generate $7 \mathrm{MPa}$ thermal stress, which is well below the tensile strength of $37 \mathrm{MPa}$.
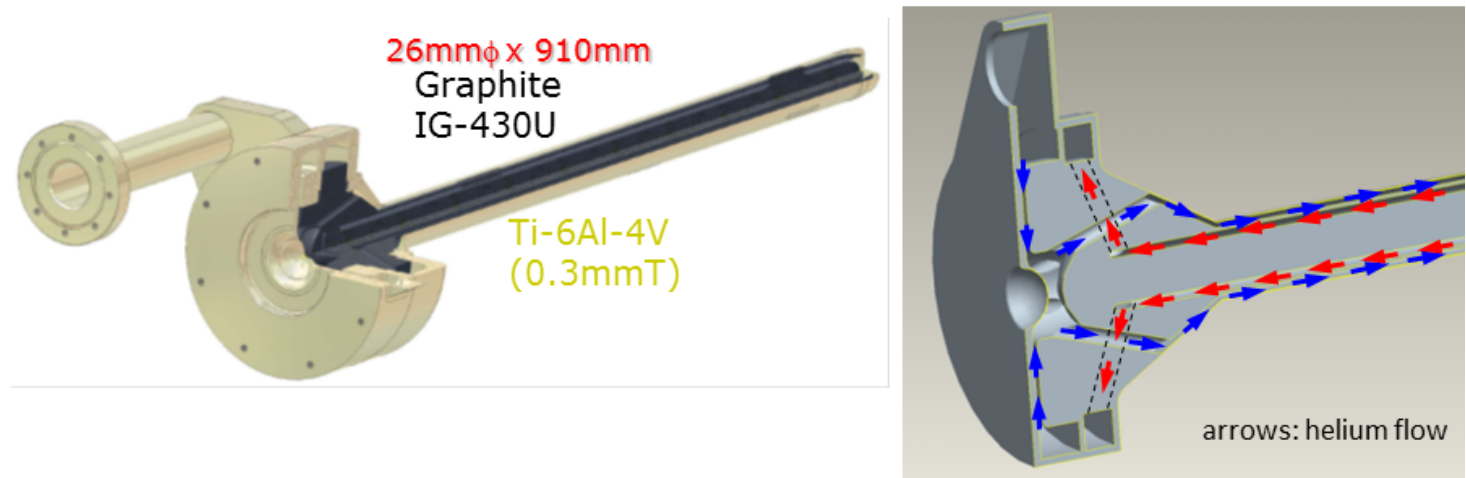

FIGURE 4. Schematic view of the T2K target.Right figure is a zoomed-up cut-view of the head part with arrows indicating the Helium gas flow for cooling.

\section{NuMI target}

The neutrino beam line at Main Injector (NuMI) also has a graphite target. It is made of $4720 \mathrm{~mm}$-long $6.4 \mathrm{~mm}$ wide separated fins and total thickness is $954 \mathrm{~mm}$. In the low-energy (LE) target configuration adopted for MINOS the target is inserted into horn1, and the graphite fins are cooled by water pipes attached to the top and bottom edge 

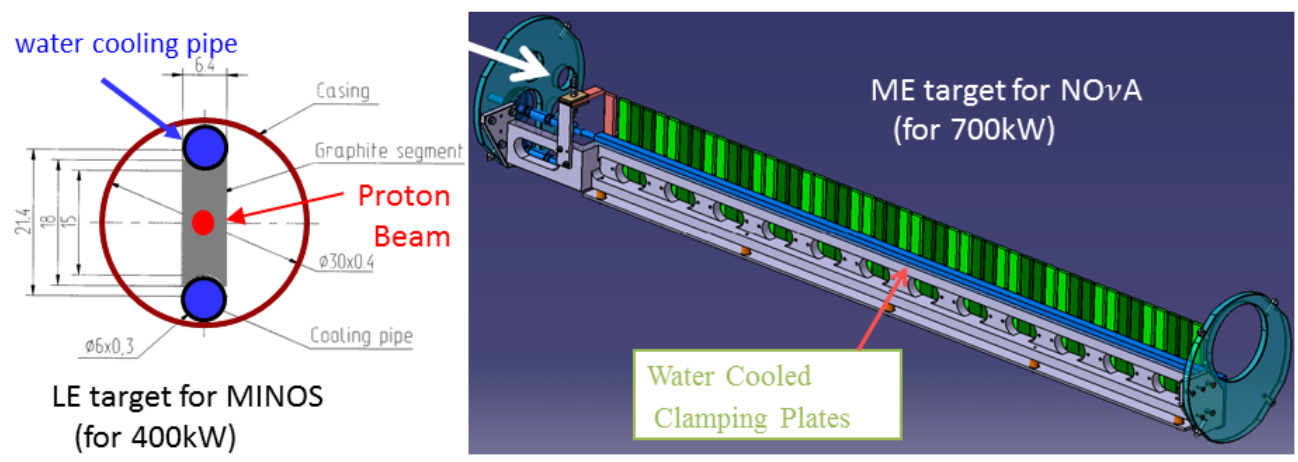

FIGURE 5. Schematic view of the NuMI target. Left is the cross section of the LE target for MINOS, right is the ME target for NOvA.

of fins. The acceptable beam power is $400 \mathrm{~kW}$. The medium energy (ME) operation for NOvA, in which the target is placed upstream the horn1, arrows wider space around the target for cooling. The new ME target upgraded for NOvA is cooled by the water-cooled clamping plates and can accept a $700 \mathrm{~kW}$ beam. Schematic views of the LE target for MINOS and ME target for NOvA are shown in Fig.5.

\section{Ideas for future}

The LBNF is aimed for running at a $1 \sim 3 \mathrm{MW}$. By enlarging the cross-sectional dimension from the NuMI LE target and beam spot, it is expected that the target can accept $1.2 \mathrm{MW}$ beam. For higher beam power such as $2.5 \mathrm{MW}$, one of the ideas is to use a number of beryllium spheres cooled by Helium gas.

For the ESS neutrino facility, a parallel four target-and-horn systems configuration is being considered to reduce the load to each system.

\section{Electromagnetic Horn}

The electromagnetic horn is made of coaxial aluminum tubes which are connected at one end as shown in Fig.6. By flowing high electrical current, toroidal magnetic field is produced. The strength of the field is given by $B[\mathrm{~T}]=\frac{I[\mathrm{kA}]}{5 r[\mathrm{~mm}]}$, where $I$ is the current and $r$ is the radial position. With a typical operation current of $250 \mathrm{kA}$, the magnetic field strength is $1.8 \mathrm{~T}$ at $r=28 \mathrm{~mm}$. The beam power acceptable to horns is limited by cooling capacity and thermal stress. The horn conductors are cooled by spray water, which causes issues of productions of hydrogen, Tritium, NOx etc. The horns designed so far was for $<\sim 700 \mathrm{~kW}$ and development is necessary to go beyond that.

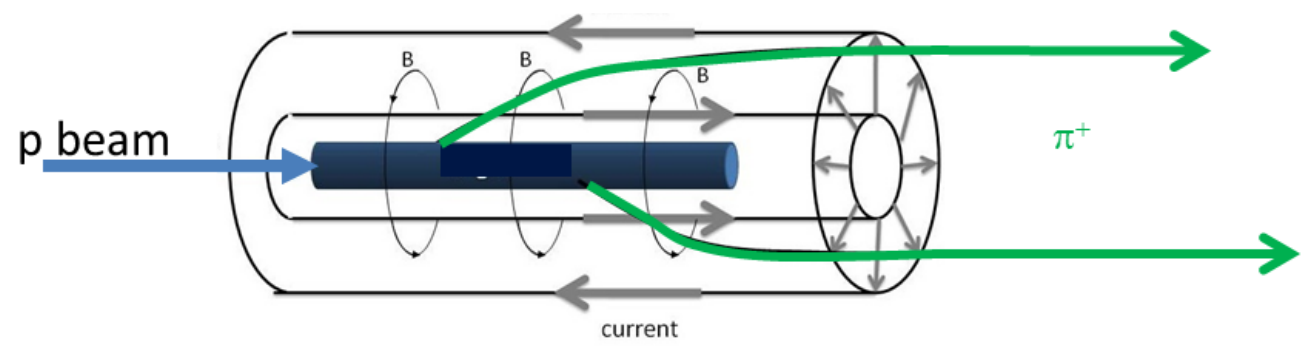

FIGURE 6. Schematic view of the electromagnetic horn. 


\section{Decay Volume and Beam Dump}

Since the decay volume and beam dump cannot be upgraded later once they are exposed to significant amount of radiation, those of J-PARC was designed for the $3 \mathrm{MW}$ beam power. The iron wall of the decay pipe is water-cooled. The beam dump core is made of graphite. Entire volume of the secondary beamline is filled with Helium to reduce the pion absorption and also to reduce NOx production.

The LBNF facility is currently being designed and it's target is $2.3 \mathrm{MW}$ The target chase will be filled with air and cooled by either air or water. The decay volume will be filled with helium, but the wall will be cooled by air. The beam dump core is made of Aluminum and cooled by water.

\section{SUMMARY}

High-intensity proton accelerator complex enabled long baseline neutrino oscillation experiments with a precisely controlled neutrino beam. The beam power so far achieved is a few hundred $\mathrm{kW}$ with enormous efforts of accelerator physicists and engineers. However, to fully understand the lepton mixing structure, MW-class accelerators are desired. The current limitation comes from the beam loss at early stages of the accelerator complex. Higher repetition rate or higher injection energy at early stages are necessary to drastically break the current limit. Plans of upgrade of the existing facilities or new facilities are presented in this paper.

Secondary beamline equipments are exposed to enormous amount of radiation, which causes high and repeated thermal stress, heat load, radiation damage, high radio-activation and so on. Carefully designing and continuous efforts enabled secondary beamline equipments to steadily accept a few hundred $\mathrm{kW}$ proton beam. Further new ideas and development are necessary o go beyond $\sim 1 \mathrm{MW}$.

\section{ACKNOWLEDGMENTS}

I would like to acknowledge C. Densham, F. Naito, T. Koseki, A. Marchionni and A. Rubbia to give me information on various neutrino facilities during their busy time.

\section{REFERENCES}

1. S. E. Kopp, arXiv:0709.2737 [hep-ex]. 